\title{
Chondrogenic differentiation of human umbilical cord blood-derived mesenchymal stem cells by co-culture with rabbit chondrocytes
}

\author{
PENGFEI ZHENG* , LI JU* , BO JIANG, LEI CHEN, ZHAN DONG, LI JIANG, RUFA WANG and YUE LOU \\ Nanjing Children's Hospital Affiliated to Nanjing Medical University, Nanjing, Jiangsu 210008, P.R. China
}

Received May 6, 2013; Accepted August 12, 2013

DOI: $10.3892 / \mathrm{mmr} .2013 .1637$

\begin{abstract}
The objective of the current study was to investigate the ability of human umbilical cord blood-derived mesenchymal stem cells (HUCB-MSCs) to undergo chondrogenic differentiation, by co-culture with rabbit chondrocytes. The aim was to obtain more seed cells for tissue engineering research and lay the foundation for the clinical repair of cartilage defects. The studies were performed using isolated rabbit cartilage cells and HUCB-MSCs in vitro, which were co-cultured in a 2:1 or 3:1 ratio with or without insulin-like growth factor-1 (IGF-1). Following 7 and 14 days in culture, cell morphology was observed in each group. RNA and protein were extracted to assess the expression levels of aggrecan (ACAN) and collagen type II (COL2A) using quantitative polymerase chain reaction (qPCR) and western blotting, respectively. Groups of cells that were co-cultured exhibited significantly higher expression levels of ACAN and COL2A mRNA and protein, compared with the reduced effect of IGF-1 at days 7 and 14 in culture. The addition of IGF-1 was found to potentiate these effects. Specifically, at day 7, cells co-cultured at a ratio of $2: 1$ had a greater induction of ACAN and COL2A compared with cells co-cultured at a 3:1 ratio. However, following 14 days culture, cells co-cultured at a 3:1 ratio with additional IGF-1 exhibited a greater induction of ACAN and COL2A compared with cells co-cultured at a ratio of 2:1. Human chondrocytes may be successfully induced by co-culture of HUCB-MSCs with rabbit chondrocytes, thus providing a theoretical basis to obtain seed cells with the capacity to differentiate into multiple
\end{abstract}

Correspondence to: Professor Yue Lou, Nanjing Children's Hospital Affiliated to Nanjing Medical University, 72 Guangzhou Road, Nanjing, Jiangsu 210008, P.R. China

E-mail: yuelou@hotmail.com

${ }^{*}$ Contributed equally

Key words: human umbilical cord blood-derived mesenchymal stem cells, co-culture, rabbit chondrocytes, insulin-like growth factor-1, aggrecan, collagen type II cell types, with low immunogenicity. Notably, these cells may provide a valuable resource for tissue engineering.

\section{Introduction}

Articular cartilage is an integral component of joint surfaces, however it has limited ability to repair itself and is difficult to repair once damaged (1). Previous developments in the bone tissue engineering field have provided hope for clinical repair of cartilage defects. The primary material used in tissue engineering is seed cells, which may be isolated and expanded in culture. Co-culture has been widely used in tissue engineering in previous years to induce human stem cells to differentiate into the desired cell type, typically using cells of animal origin, which secrete the appropriate cytokines (2). However, determining the optimal ratios of cell types to obtain specific cells and selecting the best type of human stem cells has been problematic. In 2005, Broxmeyer (3), first described that umbilical cord blood is rich in hematopoietic stem cells and subsequent studies have shown that these cells have the potential to differentiate into bone and cartilage tissue, similar to bone marrow mesenchymal stem cells (BMSCs) (4-6). A subsequent study established that human umbilical cord blood mesenchymal stem cells (HUCB-MSCs) were more suitable than BMSCs, as they did not trigger the immune response caused by the graft-versus-host disease following transplantation (7). Thus, HUCB-MSCs are considered to be of great value for the research and development of novel therapies (6). The aim of the current study was to isolate and co-culture HUCB-MSCs with rabbit chondrocytes in various proportions and to induce the differentiation of HUCB-MSCs into human chondrocytes. Notably, this technique provides a new method for obtaining more suitable seed cells for tissue engineering purposes and provides a theoretical basis for the clinical treatment of cartilage defects.

\section{Materials and methods}

Experimental materials. HUCB-MSCs (OriCell) were purchased from Biotechnology Co., Ltd. (Cyagen, Guangzhou, China). Newborn New Zealand white rabbits (1-3 days old) were provided by the Experimental Animal Center of Nanjing Medical University. The use of laboratory animals in 
these experiments was in accordance with the guidance and standards of the Ministry of Science and Technology of the People's Republic of China (8). Table I provides a detailed list of the reagents used in these experiments.

Culture and identification of HUCB-MSCs. HUCB-MSCs were cultured in DMEM (F-12) containing $15 \%$ fetal bovine serum, and $100 \mathrm{U} / \mathrm{ml}$ penicillin and streptomycin, respectively. Culture medium was changed on average every three days, depending on the rate of cell growth. The third generation of HUCB-MSCs was used to confirm their identity. The cells were washed twice with trypsin and analyzed for the expression of CD29 and CD34 by flow cytometry.

Isolation and culture of rabbit chondrocytes. Newborn New Zealand white rabbits (1-3 days old) were sacrificed and cartilage from the limb joints was removed under aseptic conditions. Cartilage was sliced into $1-3-\mathrm{mm}^{3}$ sections, placed in sterile tubes, washed three times with PBS and centrifuged at $80 \mathrm{x} \mathrm{g}$ for $3 \mathrm{~min}$. Chondrocytes were isolated by incubation with $2 \mathrm{~g} / 1$ trypsin for $30 \mathrm{~min}$, followed by digestion with $2 \mathrm{~g} / \mathrm{l}$ type II collagenase for 6-8 h. Digested cartilage was centrifuged at $80 \mathrm{x}$ g for $3 \mathrm{~min}$ and washed twice with culture medium. Cells were counted and transferred into a $25 \mathrm{ml}$ culture flask containing DMEM, at a seeding density of $5 \times 10^{9}$ cells $/ 1$. Isolated cells were cultured in an incubator at $37^{\circ} \mathrm{C}$ with $5 \% \mathrm{CO}_{2}$. Media was changed every two days. Isolated chondrocytes and HUCB-MSCs were randomly assigned to experimental groups for further analysis (Table II).

Analysis of chondrocyte-specific markers. Aggrecan (ACAN) and collagen type II (COL2A) were used as chondrocyte-specific markers. TRIzol was used to extract RNA from cells at days 7 and 14 of the experiment. Isolated RNA was reserve transcribed into cDNA which was used for quantitative polymerase chain reaction (qPCR). PCR products $(5 \mu \mathrm{l})$ were separated on agarose gels $(20 \mathrm{~g} / \mathrm{l})$ and visualized with ethidium bromide under ultraviolet light. Primer sequences are listed in Table III. Protein was also extracted from cells at days 7 and 14 of the experiment. Extracts of total cellular protein were used for western blotting to detect the expression of ACAN and COL2A and relative expression levels were assessed by grayscale analysis of digital images.

Statistical analysis. Data are presented as mean \pm SD. Differences among groups were assessed with SPSS version 11.0 statistical software (SPSS Inc, Chicago, IL), using an independent-samples t-test. $\mathrm{P}<0.05$ was considered to indicate a statistically significant difference.

\section{Results}

Identification of HUCB-MSCs. Third generation HUCB-MSCs were analyzed to confirm their identity (Fig. 1). Expression of CD29 (95.87\%) and CD34 (3.1\%) were confirmed by flow cytometry.

Quantification of chondrocyte-specific marker mRNA. Expression levels of ACAN and COL2A mRNA were assessed following 7 days in culture (Fig. 2). Following 7 days,
Table I. Summary of experimental reagents.

\begin{tabular}{ll}
\hline Reagents & \multicolumn{1}{c}{ Company } \\
\hline TIAN script RT kit & Tiangen, Beijing, China \\
RNA simple total-RNA kit & Tiangen, Beijing, China \\
2X Taq PCR Master mix & Tiangen, Beijing, China \\
Fetal bovine serum & Sijiqing, Hangzhou, China \\
DMEM/F12 medium & Gibco-BRL, Carlsbad, CA, USA \\
Aggrecan IgG & Abcam, Cambridge, MA, USA \\
Collagen type II protein IgG & Abcam, Cambridge, MA, USA \\
PVDF & Millipore, Billerica, MA, USA
\end{tabular}

PCR, polymerase chain reaction; PVDF, polyvinylidene fluoride.

Table II. Summary of experimental groups.

\begin{tabular}{lccc}
\hline & $\begin{array}{c}\text { Stem cell } \\
\text { suspension } \\
\left(5 \times 10^{9} / 1\right)\end{array}$ & $\begin{array}{c}\text { Chondrocyte } \\
\text { suspension } \\
\left(5 \times 10^{9} / 1\right)\end{array}$ & IGF-1 \\
\hline A, simple stem cell- & $6.0 \mathrm{ml}$ & None & $100 \mu \mathrm{g} / 1$ \\
induced & $4.0 \mathrm{ml}$ & $2.0 \mathrm{ml}$ & None \\
B, $2: 1$ co-culture & $4.5 \mathrm{ml}$ & $1.5 \mathrm{ml}$ & $100 \mu \mathrm{g} / 1$ \\
C, $2: 1$ co-culture+IGF-1 & $4.0 \mathrm{ml}$ & $2.0 \mathrm{ml}$ & None \\
D, 3:1 co-culture & $4.5 \mathrm{ml}$ & $1.5 \mathrm{ml}$ & $100 \mu \mathrm{g} / 1$ \\
E, 3:1 co-culture+IGF-1 & & & \\
\hline
\end{tabular}

IGF-1, insulin-like growth factor-1.

co-cultured cells in group D exhibited a slight increase in the expression levels of ACAN and COL2A mRNA with the addition of insulin-like growth factor (IGF-1), however, all other groups exhibited a higher expression level of ACAN and COL2A mRNA compared with the control group $(\mathrm{P}<0.05)$. In co-cultures of the two cell ratios tested, ACAN and COL2A mRNA expression increased following the addition of IGF-1 $(\mathrm{P}<0.05)$. Under the same culture conditions, the effects in groups with different ratios of cells were compared. ACAN and COL2A mRNA expression were observed to be higher in group $\mathrm{B}$ compared with group $\mathrm{D}(\mathrm{P}<0.05)$ and $\mathrm{ACAN}$ and COL2A mRNA expression were higher in group $\mathrm{C}$ compared with group $\mathrm{E}(\mathrm{P}<0.05)$.

Expression levels of ACAN and COL2A mRNA were also assessed following 14 days in culture (Fig. 3). Following 14 days, the groups containing co-cultured cells exhibited a higher expression level of ACAN and COL2A mRNA compared with the control group $(\mathrm{P}<0.05)$. However, the expression of ACAN and COL2A only increased slightly in group B, with no statistically significant difference observed $(\mathrm{P}>0.05)$, indicating that co-culture induced slightly improved chondrocyte differentiation compared with the reduced effect of IGF-1. In co-cultures of the two cell ratios, ACAN and COL2A mRNA expression increased following the addition of IGF-1 $(\mathrm{P}<0.05)$. Under the same culture condi- 
Table III. Oligonucleotide primer sets for reverse transcription-polymerase chain reaction.

\begin{tabular}{|c|c|c|c|c|}
\hline Gene & Sequence $\left(5^{\prime}-3^{\prime}\right)$ & Length, nt & $\mathrm{Tm},{ }^{\circ} \mathrm{C}$ & Size, bp \\
\hline Aggrecan-F & CACCTACAAACGCAGACTACAGA & 23 & 57.6 & 167 \\
\hline Aggrecan- $\mathrm{R}$ & AAAGCGACAAGAAGAGGACACC & 22 & 60.2 & \\
\hline COL2A-F & CAGCAAGAGCAAGGAGAAG & 19 & 53.1 & 126 \\
\hline COL2A-R & AGGCGTAGGAAGGTCATC & 18 & 51.7 & \\
\hline$\beta$-actin-F & CTTAGTTGCGTTACACCCTTTCTTG & 25 & 62.0 & 156 \\
\hline$\beta$-actin-R & CTGTCACCTTCACCGTTCCAGTTT & 24 & 64.4 & \\
\hline
\end{tabular}

COL2A, collagen type II.
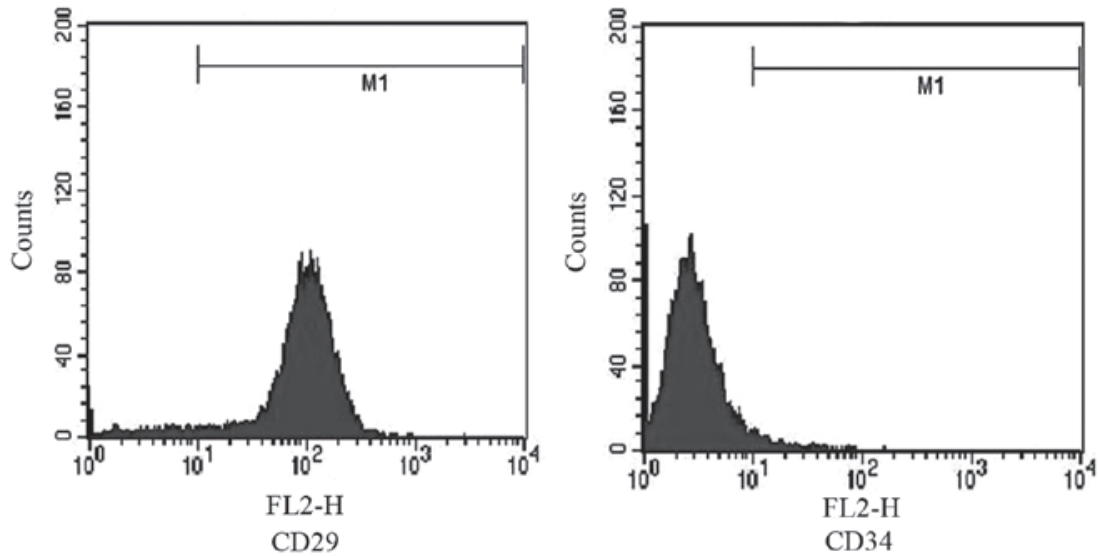

Figure 1. HUCB-MSCs cell surface markers. Third generation HUCB-MSCs were analyzed to confirm their identity by flow cytometry. Expression of CD29 and CD34 was 95.87 and 3.1\%, respectively. HUCB-MSCs, human umbilical cord blood-derived mesenchymal stem cells.

tions, the effects in the groups with different ratios of cells were compared. COL2A mRNA expression was higher in group D compared with group $\mathrm{B}(\mathrm{P}<0.05)$ and ACAN expression was higher in group $\mathrm{B}$ compared with group $\mathrm{D}$, however, this difference was not statistically significant $(\mathrm{P}>0.05)$. ACAN expression was higher in group B compared with D, however, this difference was also not statistically significant ( $\mathrm{P}>0.05)$. When groups $\mathrm{C}$ and $\mathrm{E}$ were compared, COL2A mRNA expression was higher in group $\mathrm{E}(\mathrm{P}<0.05)$ and ACAN mRNA expression was higher in group $\mathrm{C}(\mathrm{P}<0.05)$. Comparions of days 7 and 14 for groups $\mathrm{C}$ and $\mathrm{E}$ showed that ACAN mRNA expression was higher at day 14, similar to the level observed in group B.

Protein expression levels of chondrocyte-specific markers. The protein expression levels of ACAN and COL2A were assessed following 7 days in culture (Fig. 4). Following 7 days, groups containing co-cultured cells exhibited higher protein expression levels of ACAN and COL2A compared with the control group $(\mathrm{P}<0.05)$, with the exception of group $\mathrm{D}$, which indicated that IGF-1 induced improved differentiation in co-culture compared with IGF-1 alone. In co-cultures of the two cell ratios, ACAN and COL2A protein expression increased following the addition of IGF-1 $(\mathrm{P}<0.05)$. Under the same culture conditions, the effects in groups with different ratios of cells were compared. ACAN and COL2A protein expression was found to be higher in group B compared with $\mathrm{D}(\mathrm{P}<0.05)$ and ACAN and COL2A protein expression were higher in group $\mathrm{C}$ compared with $\mathrm{E}(\mathrm{P}<0.05)$.

The protein expression levels of ACAN and COL2A were also assessed following 14 days in culture (Fig. 5). Following 14 days, the groups containing co-cultured cells exhibited a higher expression level of ACAN and COL2A protein compared with the control group $(\mathrm{P}<0.05)$. In co-cultures with the two cell ratios tested, ACAN and COL2A protein expression increased following the addition of IGF-1 $(\mathrm{P}<0.05)$. Under the same culture conditions, the effects in groups with different ratios of cells were compared. COL2A protein expression was found to be higher in group D compared with $\mathrm{B}(\mathrm{P}<0.05)$ and $\mathrm{ACAN}$ expression was higher in group $\mathrm{B}$ compared with $\mathrm{D}$, however, this difference was not statistically significant $(\mathrm{P}>0.05)$. ACAN expression was higher in group $\mathrm{B}$ compared with group $\mathrm{D}$, however, this difference was not statistically significant $(\mathrm{P}>0.05)$. When groups $\mathrm{C}$ and $\mathrm{E}$ were compared, COL2A protein expression was observed to be higher in group $\mathrm{E}$, but this difference was not statistically significant $(\mathrm{P}>0.05)$. However, ACAN protein expression levels were significantly higher in group $\mathrm{E}$ compared with group $\mathrm{C}(\mathrm{P}<0.05)$.

\section{Discussion}

In previous years, bone tissue engineering has been applied for the repair of articular cartilage defects in basic and clinical 

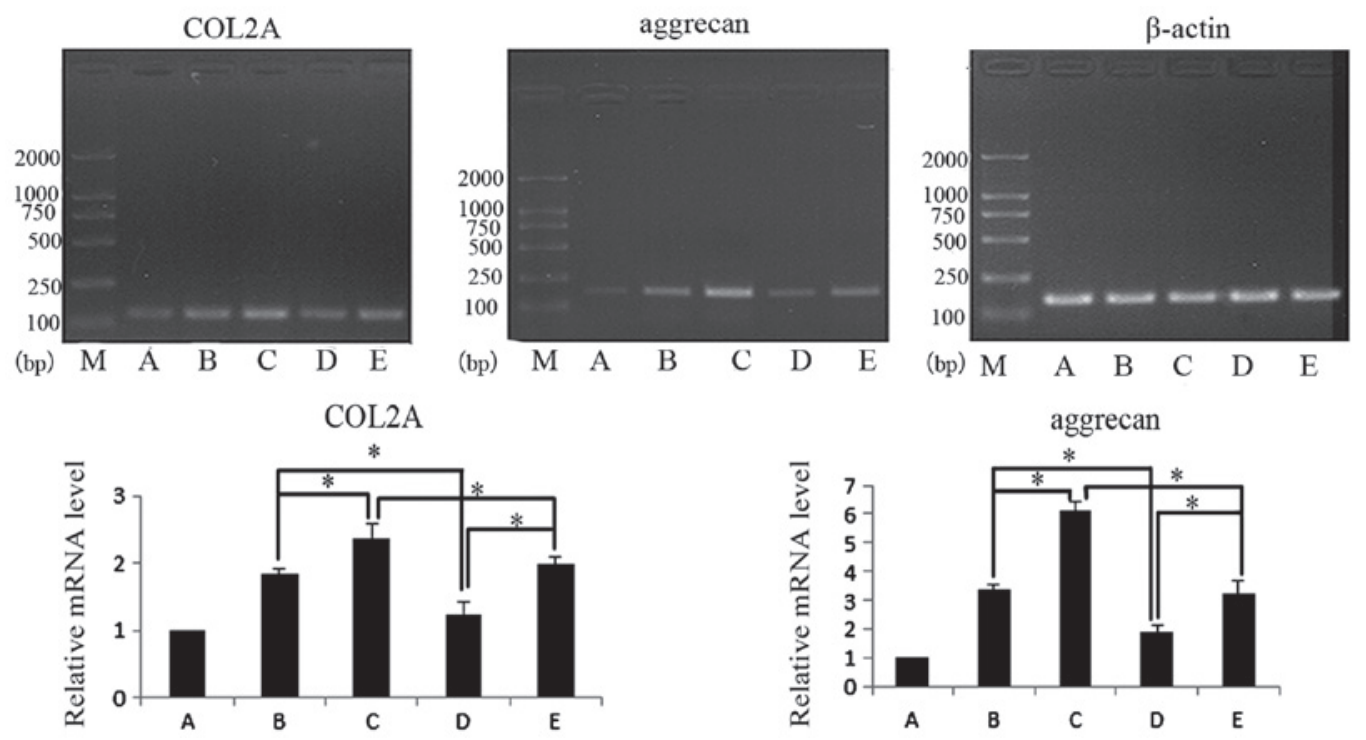

Figure 2. Electrophoretogram and gradation analysis chart of ACAN, COL2A and $\beta$-actin mRNA expression in human umbilical cord blood-derived mesenchymal stem cells co-cultured with rabbit chondrocytes at day 7. A) Simple stem cell-induced, B) 2:1 co-culture, C) 2:1 co-culture + insulin-like growth factor-1 (IGF-1), D) 3:1 co-culture and E) 3:1 co-culture + IGF-1. ${ }^{*} \mathrm{P}<0.05$ indicates significant differences between compared groups.. ACAN, aggrecan; COL2A, collagen type II.

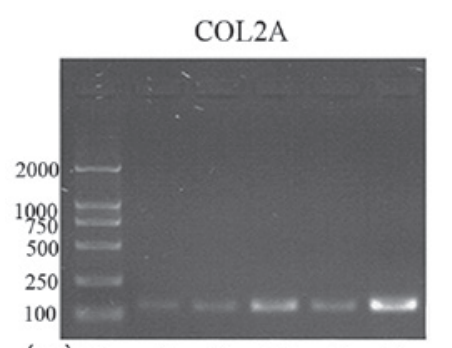

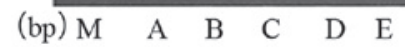

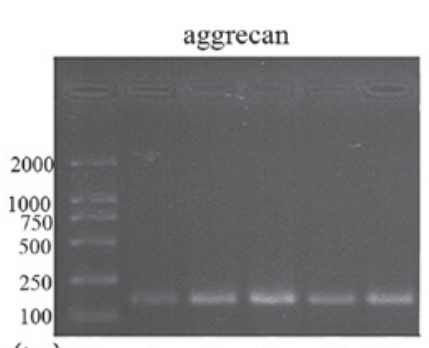

(bp) $\begin{array}{llllll}M & \text { A } & \text { B } & \text { C } & \text { D } & \text { E }\end{array}$

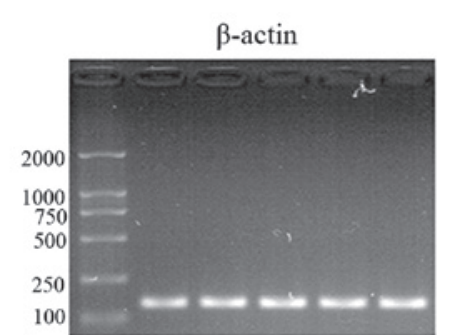

(bp) $\begin{array}{llllll}\mathrm{M} & \mathrm{A} & \mathrm{B} & \mathrm{C} & \mathrm{D} & \mathrm{E}\end{array}$
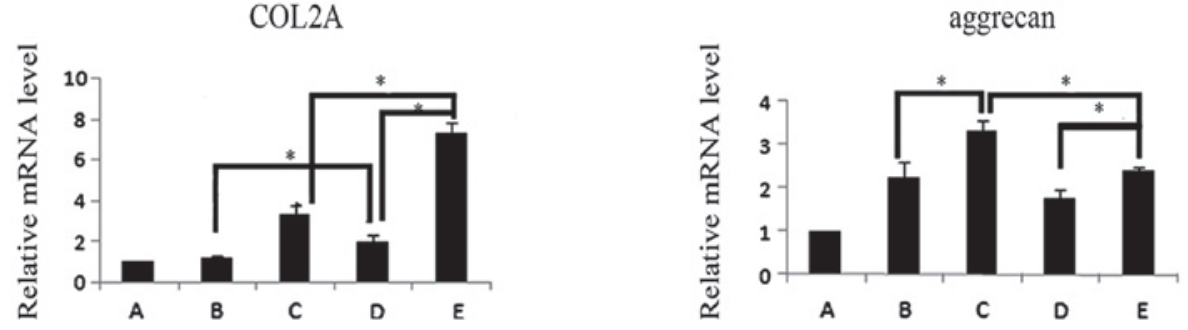

Figure 3. Electrophoretogram and gradation analysis chart of ACAN, COL2A and $\beta$-actin mRNA expression in human umbilical cord blood-derived mesenchymal stem cells co-cultured with rabbit chondrocytes at day 14. A) Simple stem cell-induced, B) 2:1 co-culture, C) 2:1 co-culture + insulin-like growth factor-1 (IGF-1), D) 3:1 co-culture and E) 3:1 co-culture + IGF-1. " $\mathrm{P}<0.05$. $\mathrm{P}<0.05$ indicates significant differences between compared groups. ACAN, aggrecan, COL2A, collagen type II.

research and has gained increasingly wider attention from investigators and clinicians. Studies have shown that co-culturing chondrocytes with BMSCs and the use of chondrocyte paracrine secretions, may help to regulate the differentiation of mesenchymal stem cells into chondrocytes and may significantly reduce the requirements of chondrocytes. This has provided a practical method to solve the insufficient source of chondrocytes for tissue engineering (2). Results of recent studies have shown that animal-derived cartilage cells secrete the same cytokines and induce human stem cells to differentiate into chondrocytes $(7,9)$. This observation was a major breakthrough in tissue engineering and allow the possibility of using animal cells in co-culture with human stem cells to promote chondrogenic differentiation.
HUCB-MSCs have numerous advantages compared with BMSCs, including convenient collection, easy separation and preservation, reduced immunogenicity, the absence of tumor cell contamination, a lower risk of latent virus and pathogenic microorganism infection and transmission and less ethical controversy (10). IGF-1, transforming growth factor- $\beta$ (TGF- $\beta$ ), bone morphogenetic protein and fibroblast growth factor 2 have all been shown to induce bone and cartilage differentiation in HUCB-MSCs, similar to BMSCs (11-13). The ability of co-culture of HUCB-MSCs and chondrocytes to obtain large numbers of chondrocytes has not been reported in previous literature and the majority of stem cells and chondrocytes are co-cultured at a ratio of 1:1 or 2:1. As HUCB-MSCs may be obtained more readily than chondrocytes, inducing more 

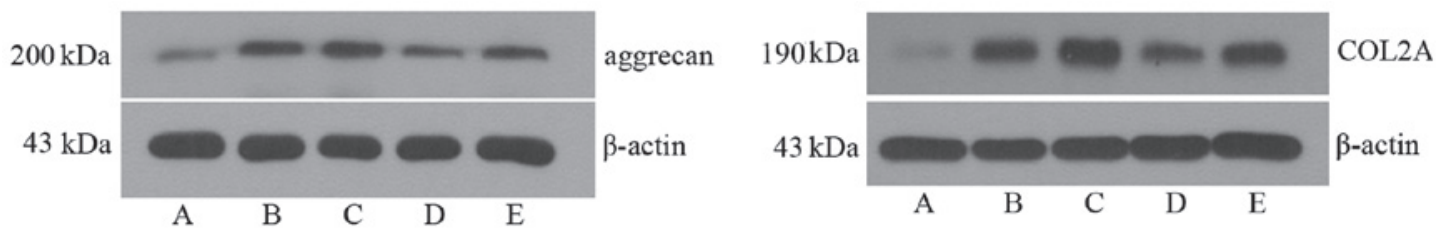

COL2A
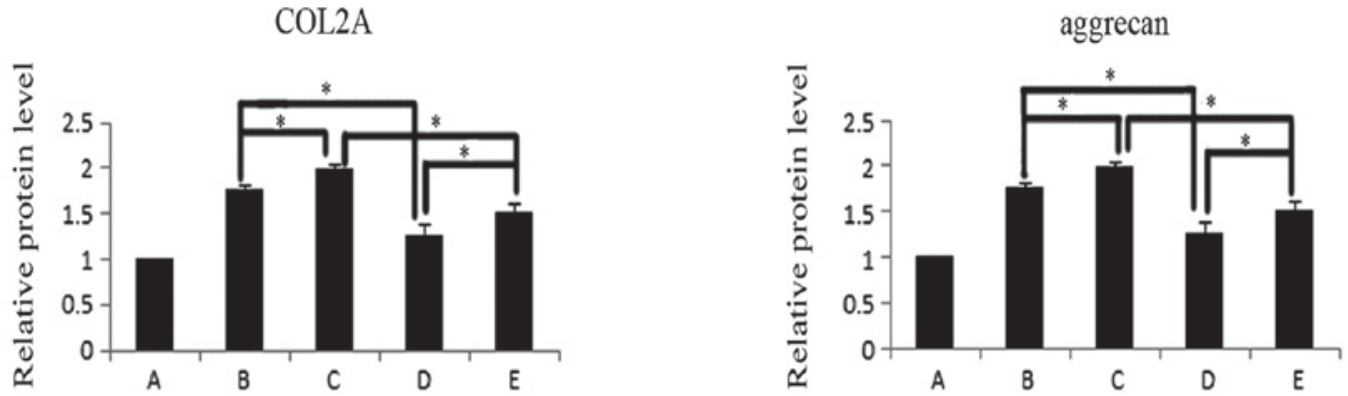

Figure 4. Electrophoretogram and gradation analysis chart of ACAN, COL2A and $\beta$-actin protein expression in human umbilical cord blood-derived mesenchymal stem cells co-cultured with rabbit chondrocytes at day 7. A) Simple stem cell-induced, B) 2:1 co-culture, C) 2:1 co-culture + insulin-like growth factor-1 (IGF-1), D) 3:1 co-culture and E) 3:1 co-culture + IGF-1. *P<0.05. ACAN, aggrecan, COL2A, collagen type II.
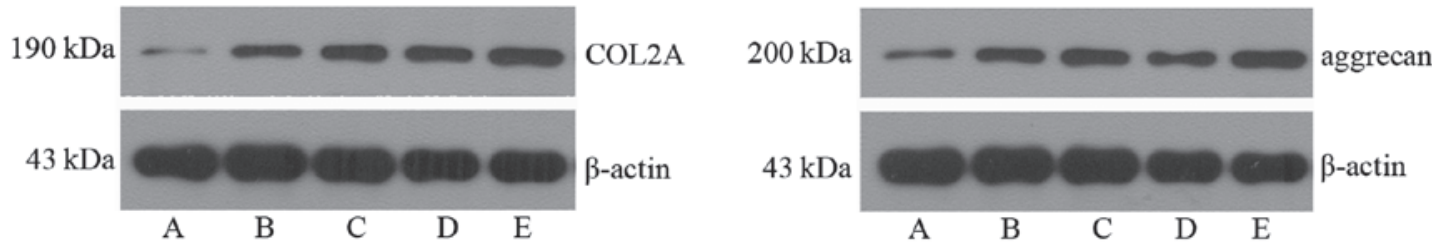

COL2A
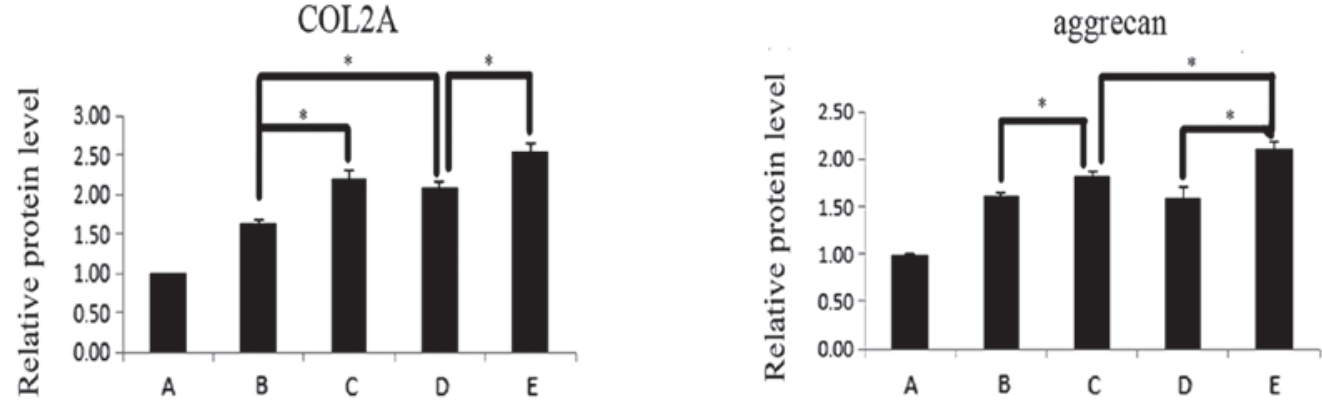

Figure 5. Electrophoretogram and gradation analysis chart of ACAN, COL2A and $\beta$-actin protein expression in human umbilical cord blood-derived mesenchymal stem cells co-cultured with rabbit chondrocytes at day 14. A) Simple stem cell-induced, B) 2:1 co-culture, C) 2:1 co-culture + insulin-like growth factor-1 (IGF-1), D) 3:1 co-culture and E) 3:1 co-culture + IGF-1. ${ }^{*}$ P<0.05. ACAN, aggrecan, COL2A, collagen type II.

chondrogenic differentiation of stem cells with fewer chondrocytes is likely to be the focus of future studies. In the current study, HUCB-MSCs and rabbit chondrocytes were co-cultured at a ratio of 2:1 and 3:1, in the presence or absence of IGF-1, to assess the feasibility of using fewer chondrocytes to induce HUCB-MSC differentiation and thus, provide an improved source of cells for tissue engineering.

To determine the relative levels of HUCB-MSC differentiation into chondrocytes, the expression of the markers of chondrocyte differentiation, ACAN and COL2A, were compared at the protein and mRNA levels. Primers specific to human transcripts and human-specific antibodies were used to exclude the possibility of interference from endogenous rabbit chondrocytes. The results showed that co-culture with rabbit chondrocytes induced greater differentiation of HUCB-MSCs into chondrocytes compared with a reduced effect of IGF-1.
This observation was confirmed by higher mRNA and protein levels of ACAN and COL2A. The addition of IGF-1 to the culture medium increased chondrocyte differentiation. Although cells co-cultured at a ratio of 2:1 exhibited higher rates of differentiation compared with cells co-cultured at a $3: 1$ ratio, the addition of IGF-1 yielded superior results. This indicates that the addition of cytokines, specifically IGF-1, induces HUCB-MSC differentiation in the presence of fewer chondrocytes.

In conclusion, the co-culture of HUCB-MSCs and rabbit chondrocytes induces the differentiation of HUCB-MSCs into human chondrocytes. The current study provides evidence of a principle for a theoretical source of multi-potent stem cells with low immunogenicity, yielding a rich source of seed cells for tissue engineering. Although seed cells are an important consideration in tissue engineering, other factors, including 
the tissue scaffold and micronutrient environment are also significant. Accordingly, to obtain the desired tissue with the maximum efficiency, exploration of total culture time (14), the use of growth factors, including IGF-1 and TGF- $\beta$ (15), hierarchical co-culture, conditioned medium and joint specific scaffolds (16), requires further study.

\section{Acknowledgements}

This study was supported by a grant from the Health Bureau of Nanjing (no. QYK10163). The authors would like to thank Yue Lou, for her guidance in the writing of this manuscript.

\section{References}

1. Ochi M: Clinical results of transplantation of tissue-engineered and future direction of cartilage repair-novel approach with minimally invasive procedure. Yonsei Med J 45: 74-74, 2004.

2. Tsuchiya K, Chen G, Ushida T, Matsuno T and Tateishi T: The effect of coculture of chondrocytes with mesenchymal stem cells on their cartilaginous phenotype in vitro. Mater Sci Eng C Mater Biol Appl 24: 391-396, 2004.

3. Broxmeyer HE: Biology of cord blood cells and future prospects for enhanced clinical benefit. Cytotherapy 7: 209-218, 2005.

4. Zhang X, Hirai M, Cantero S, Ciubotariu R, Dobrila L, Hirsh A, Igura K, Satoh H, Yokomi I, Nishimura T, et al: Isolation and characterization of mesenchymal stem cells from human umbilical cord blood: reevaluation of critical factors for successful isolation and high ability to proliferate and differentiate to chondrocytes as compared to mesenchymal stem cells from bone marrow and adipose tissue. J Cell Biochem 112: 1206-1218, 2011.

5. Feng X, Tian S, Sun K, Zhang J, Zhang C, Liu S, Zhou M and Lü J: Effect of platelet lysate on chondrogenic differentiation of human umbilical cord derived mesenchymal stem cells in vitro. Zhongguo Xiu Fu Chong Jian Wai Ke Za Zhi 25: 1250-1255, 2011 (In Chinese).
6. Ghen MJ, Roshan R, Roshan RO, et al: Potential clinical applications using sten cells derived from human umbilical cord blood. Reprod Biomed Online 13: 562-572, 2006.

7. Meretoja VV, Dahlin RL, Kasper FK and Mikos AG: Enhanced chondrogenesis in co-cultures with articular chondrocytes and mesenchymal stem cells. Biomaterials 33: 6362-6369, 2012.

8. The Ministry of Science and Technology of the People's Republic of China. Guidance suggestion of caring laboratory animals. http:// www.most.gov.cn/fggw/zfwj/zfwj2006/200609/t20060930_54389. $\mathrm{htm}$. Accessed September 30, 2006 (In Chinese).

9. Wu L, Prins HJ, Helder MN, van Blitterswijk CA and Karperien M: Trophic effects of mesenchymal stem cells in chondrocyte co-cultures are independent of culture conditions and cell sources. Tissue Eng Part A 18: 1542-1551, 2012.

10. Gennery AR and Cant AJ: Cord blood stem cell tansplantation in primary immune deficiencies. Curr Opin Allergy Clin Immunol 7: 528-534, 2007.

11. Puetzer JL, Petitte JN and Loboa EG: Comparative review of growth factors for induction of three-dimensional in vitro chondrogenesis in human mesenchymal stem cells isolated from bone marrow and adipose tissue. Tissue Eng Part B Rev 16: 435-444, 2010.

12. Weiss S, Hennig T, Bock R, Steck E and Richter W: Impact of growth factors and PTHrP on early and late chondrogenic differentiation of human mesenchymal stem cells. J Cell Physiol 223: 84-93, 2010.

13. Djouad F, Bony C, Canovas F, Fromigué O, Rème T, Jorgensen C and Noël D: Transcriptomic analysis identifies Foxo3A as a novel transcription factor regulating mesenchymal stem cell chrondrogenic differentiation. Cloning Stem Cells 11: 407-416, 2009.

14. Chang Q, Cui W-D and Fan W-M: Co-culture of chondrocytes and bone marrow mesenchymal stem cells in vitro enhances the expression of cartilaginous extracellular matrix components. Braz J Med Biol Res 44: 303-310, 2011.

15. Ertan AB, Yilgor P, Bayyurt B, et al: Effect of double growth factor release on cartilage tissue engineering. J Tissue Eng Regen Med 7: 149-160, 2013.

16. Kang N, Liu X, Guan Y, Wang J, Gong F, Yang X, Yan L, Wang Q, Fu X, Cao Y and Xiao R: Effects of co-culturing BMSCs and auricular chondrocytes on the elastic modulus and hypertrophy of tissue engineered cartilage. Biomaterials 33: 4535-4544, 2012. 